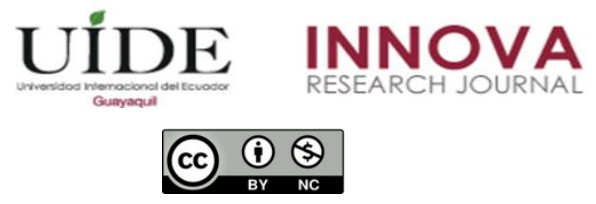

INNOVA Research Journal, ISSN 2477-9024

(Septiembre-Diciembre 2019). Vol. 4, No.3.2 pp. 15-39

DOI: https://doi.org/10.33890/innova.v4.n3.2.2019.1085

URL: http://revistas.uide.edu.ec/index.php/innova/index

Correo: innova@uide.edu.ec

\title{
Aplicación de la estrategia del employer engagement para mejorar la comunicación interna en una empresa privada
}

\section{Application of the employer engagement strategy to improve internal communication in a private company}

Julio Manuel Gonzalez Fernandez

https://orcid.org/0000-0002-8369-9659

Ester Yazmin Quispe Merlo

https://orcid.org/0000-0001-7049-0330

Jose Omar Casallo Durand

https://orcid.org/0000-0002-3292-8482

Rodrigo Edgardo Camacho Urbina

https://orcid.org/0000-0002-2268-0962

Universidad Norbert Wiener, Perú

Autor para correspondencia: julio31189@hotmail.com; yazmin_quime@ hotmail.com; casallodurand2093@gmail.com; rodrigoedcu1@gmail.com

Fecha de recepción: 13 de julio del 2019 - Fecha de aceptación: 14 de noviembre del 2019

\section{Resumen}

El objetivo fue proponer una estrategia para mejorar la comunicación interna en una empresa privada. Con una metodología basada en el enfoque mixto, tipo proyectivo, nivel comprensivo, método inductivo-deductivo. Se obtuvo como resultados problemas de comunicación interna, cuya situación causa en los empleados un bajo interés en la resolución de problemas de gestión y poca eficiencia en el cumplimiento de sus funciones, en cuanto a la identidad corporativa, se observó que los empleados no tienen una buena imagen de la organización, además, los colaboradores no participan en actividades colectivas y no aportan ideas para mejorar el estilo de trabajo y la adaptación en la empresa, así mismo, hay un mal uso de los canales de comunicación, generando que tanto el compromiso como la motivación se vean disminuidos, afectando negativamente los resultados. Se propuso aplicar la estrategia del Employer engagement, cuyas directrices radican en realizar la técnica del Employee Branding y la gestión de recursos humanos 2.0, ambas buscan convertir a los empleados en la cara visible de la empresa, optimizar la identidad corporativa de la organización, mejorar la gestión del personal y su rendimiento, crear estrategias de comunicación mejorando el valor emocional del trabajo y la fidelización.

Palabras Claves: Employer engagement; personal; motivación; branding; identidad corporativa

\footnotetext{
Abstract

The objective was to propose a strategy to improve internal communication in a private company. With a methodology based on the mixed approach, projective type, comprehensive level, inductive-deductive method. Internal communication problems were obtained as results, whose situation causes low interest in the resolution of management problems and low efficiency in the 
fulfillment of their functions, in terms of corporate identity, it was observed that employees do not have a Good image of the organization, in addition, employees do not participate in collective activities and do not contribute ideas to improve the work style and adaptation in the company, likewise, there is a misuse of communication channels, generating both the commitment as the motivation is diminished, negatively affecting the results. It was proposed to apply the Employer engagement strategy, whose guidelines lie in performing the Employee Branding technique and the management of human resources 2.0, both seek to turn employees into the visible face of the company, optimize the corporate identity of the organization, improve Personnel management and performance, create communication strategies improving the emotional value of work and loyalty. Key words: Employer engagement; personal; motivation; branding; corporate identity

\section{Introducción}

La problemática en este artículo es realizar un análisis del estado en que se encuentra la comunicación interna en una empresa privada y conocer que situaciones se presentan durante el trabajo diario de los empleados. De acuerdo con la real academia de la lengua española, la comunicación se define como una acción de transmitir un mensaje, determina la comunicación como un accionar donde interviene la retroalimentación del mensaje. Así mismo, la comunicación interna tiene como fin brindar alcances y objetivos comunes en el empleado y afianzarlo con las decisiones tomadas por los directivos (Berceruelo, 2011). Por otro lado, la comunicación también es parte del éxito de la empresa cuando la optimización del trabajo y los estímulos entren en congruencia con los resultados que se esperan (Tessi, 2013).

Aceptado como un factor importante para lograr objetivos y metas en una organización, se han establecidos criterios y modelos de gestión para optimizar la comunicación en los grupos de trabajo, y así mismo su resultado proviene del estudio realizado por distintos autores, en ese sentido es necesario recopilar un marco de teorías que sigan a la variable en estudio.

Contamos entonces con la influencia que existe dentro del comportamiento de las personas, y los estímulos que lo aquejan en un ambiente social del día a día, expresado en la teoría del campo de fuerzas (Fernández y Puente, 2009). También debemos tener en cuenta los objetivos enfocados netamente en las responsabilidades de cada persona, como lo expresa la teoría de la administración por objetivos (Chiavenato, 2014) donde se dictan los lineamientos para realizar cada función, y, la teoría del comportamiento humano (Chiavenato, 2014), un concepto amplio que habla sobre la influencia en las personas y capacidad para responder a estímulos que fortalezcan a la organización. Así mismo, podemos referirnos al logro de objetivos dependientes de la personalidad de las personas encargadas de ejecutarlos, como lo indica la teoría de las necesidades de Mc Clelland, existe una necesidad por encontrar resultados basándose en el control de la necesidad de poder en los inviduos (Rodríguez, 2018). Y, como punto de inflexión, la teoría de las expectativas, donde se le da importancia en las actitudes y creencias para el futuro en cada individuo (Porter, Hackman y Lawler, 1975)

Y, como aspecto importante en la comunicación interna, se encuentra el liderazgo, conocido en las organizaciones como un modelo de empoderamiento para los empleados, indispensable para transmitir distintos factores a los grupos de trabajo (Iturrioz, 2017) 
Otro aspecto importante es la motivación, determinada por factores derivados en necesidades extrínsecas, y de autorrealización donde los logros juegan un papel importante para una persona dentro de un equipo de trabajo (Herzberg, 1968).

Luego tenemos la identidad corporativa, y se refiere a la imagen que tiene el empleado de su centro de trabajo, y el nivel de identificación que siente, derivando en el sentido de pertenencia hacia la organización y como lo representan (Currás, 2010).

Finalmente, el empowerment, de gran importancia para la organización, que se trata del poder que obtienen los trabajadores en la realización de trabajo para obtener responsabilidades y aspectos de personalidad y niveles de mando (Terry, 1996).

La investigación se justifica en las estrategias que se pueden proponer para solucionar los problemas de la empresa y así mismo, mejorar la comunicación interna en favor de los empleados.

Su importancia radica en los conocimientos nuevos que se pueden aportar en la investigación y reforma de los recursos humanos en el Perú y cuyos mecanismos pueden ayudar a cualquier empresa.

Para un mayor enriquecimiento del marco teórico se consultaron investigaciones anteriores como la de Preciado y Etayo (2014), donde se atribuyen tipos y estilos de liderazgo al tema de la comunicación, la investigación realizada por Cukier (2011), donde los resultados revelaron problemas relacionados a la red de información y como esta puede ser superada a partir del dialogo entre los empleados. Rodriguez (2009) indicó que toda organización es el resultado de un dialogo significativo y su propuesta en estrategias. Moreno (2015) el autor concluyó que existe un efecto en la organización comunicacional a partir de la estructura y las diferentes ramas que comprende la empresa. Armas (2014) establece un diseño descriptivo para interpretar variables y procedimientos. Garcia (2017) demostró la importancia de los recursos humanos como parte del sistema para lograr objetivos. Gutiérrez (2017) identificó la motivación y la integración como factores de importancia para ser trabajados en la organización. Condor (2018) identificó una mala comunicación interna en la empresa estudiada. Peiro y Bresó (2012) los autores identificaron facetas del modelo amigo. Pineda (2007) indicó que existe una relación entre el compromiso y el sentimiento de permanencia en el trabajo.

\section{Materiales y Métodos}

El proyecto de investigación se realizó con un enfoque mixto, utilizando el análisis cuantitativo y cualitativo, con el objetivo de recopilar datos y obtener resultados de mayor importancia y envergadura del problema; el tipo fue proyectivo, puesto que se busca proponer una alternativa de solución a las dificultades que se encuentren. El nivel fue comprensivo y el método inductivo-deductivo.

Las sub categorías de estudio fueron la motivación, identidad corporativa y empowerment. La entrevista se realizó con expertos en materia de recursos humanos y se recopilaron datos utilizando un instrumento aplica a los empleados de la empresa privada. Para el análisis de los datos cualitativos, se realizó el proceso de triangulación usando el software Atlas- 
ti y para los datos cuantitativos se usó el software SPSS, que agrupa datos utilizando histogramas y el análisis de Pareto para determinar los aspectos críticos en la organización.

Con el objetivo de analizar la comunicación interna en la empresa privada se utilizó una encuesta constituida por 16 ítems, desagregada en 3 sub categorías y cada una se constituyó en diferentes indicadores, tal como lo muestra la tabla 1.

\section{Tabla. 1}

Matriz de Operacionalización de la variable comunicación interna

\begin{tabular}{llll}
\hline Sub categoría & Indicador & Ítem & Escala \\
\hline \multirow{2}{*}{ Motivación } & $\begin{array}{l}\text { Motivación Intrínseca } \\
\text { Motivación Extrínseca } \\
\text { Comunicación }\end{array}$ & $1-6$ & \\
& & & \\
& Percepción & & Nunca \\
Identidad corporativa & Sentido de pertenencia & $7-12$ & Casi nunca \\
& Participación & & Casi siempre \\
& Actitud & & Siempre \\
& Toma de decisiones & $13-16$ & \\
Empowerment & Personalidad & & \\
\hline
\end{tabular}

Fuente: elaboración propia.

Para poder obtener los datos, se tuvo como población, que se refiere a un grupo constituido por un conjunto de elementos que poseen características similares (Hurtado, 2000), a un total 40 empleados en la organización. También, es necesario que se posea una muestra, que, según autores, se refiere a un subgrupo dentro de la población que se vuelve un sistema representativo de dicha población (Hernández, Fernandez y Baptista, 2014). Sin embargo dado el tamaño reducido de la población, se optó por utilizar el total de los 40 empleados.

Al ser una investigación mixta, el análisis cuantitativo se trabaja en un sentido sistemático de los datos numéricos y procedimientos interpretativos (Monjé, 2011).

\section{Resultados}

El análisis cuantitativo se analizó teniendo como base a cada una de las subcategorías y cada uno de los ítems que la forman, la sub categoría Motivación, formada por los ítems 1 al 6, como lo muestra la figura 1 , arrojó los siguientes resultados. 


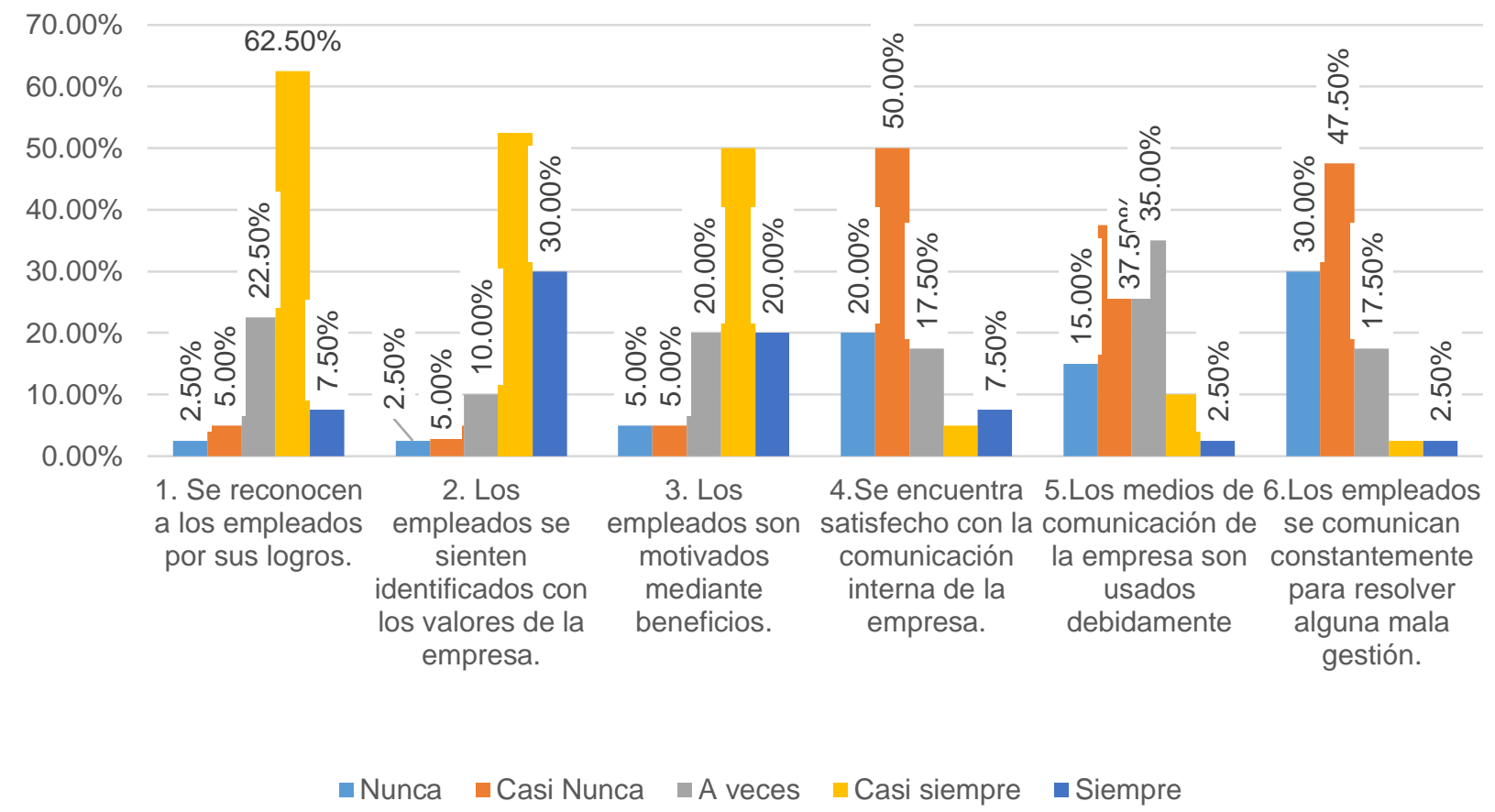

Figura 1. Frecuencias de la sub categoría Motivación (Fuente: elaboración propia)

En la motivación, se analizaron los detalles más críticos, donde, $87.5 \%$ reveló que los empleados no se encuentran satisfechos con el nivel comunicacional de la organización, evidenciando que no se usan adecuadamente los canales para enviar mensajes correctos, $87.5 \%$ indicó que los empleados se encuentran limitados en el uso de herramientas de comunicación y no pueden usarlas adecuadamente, $95 \%$ indicó que los empleados no realizan actividades para corregir problemas de la gestión, resaltado la poca eficiencia e importancia que tienen por la solución de las dificultades.

La segunda sub categoría, identidad corporativa, formada por los ítems 7 al 12, como lo muestra la figura 2 , tiene los siguientes resultados. 


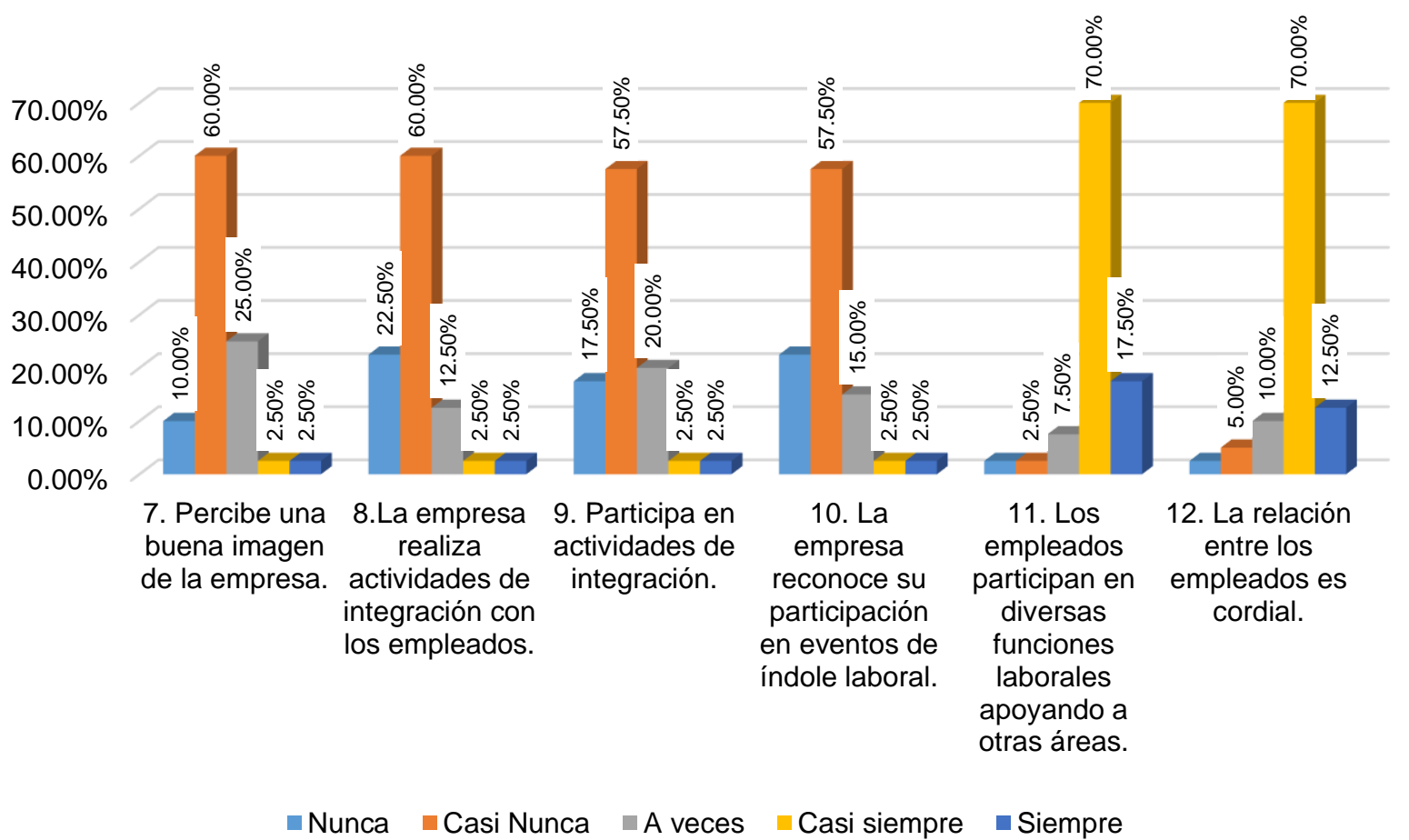

Figura 2. Frecuencias de la sub categoría identidad corporativa (Fuente: elaboración propia)

En la identidad corporativa, se analizaron los factores más críticos, donde, 95\% reveló no tener una buena imagen de la empresa por el poco apoyo que brinda a los trabajadores, $95 \%$ indica que los trabajadores no se integran adecuadamente y no trabajan en equipo, 95\% reveló que la empresa no invierte en realizar actividades que mejores el sentido de pertenencia de los trabajadores, y, 95\% evidencia que la empresa no muestra interés en que los empleados participen de eventos relacionados con la compañía.

La tercera sub categoría, recursos disponibles, formada por los ítems 13 al 16, como lo muestra la figura 3 , tiene los siguientes resultados. 


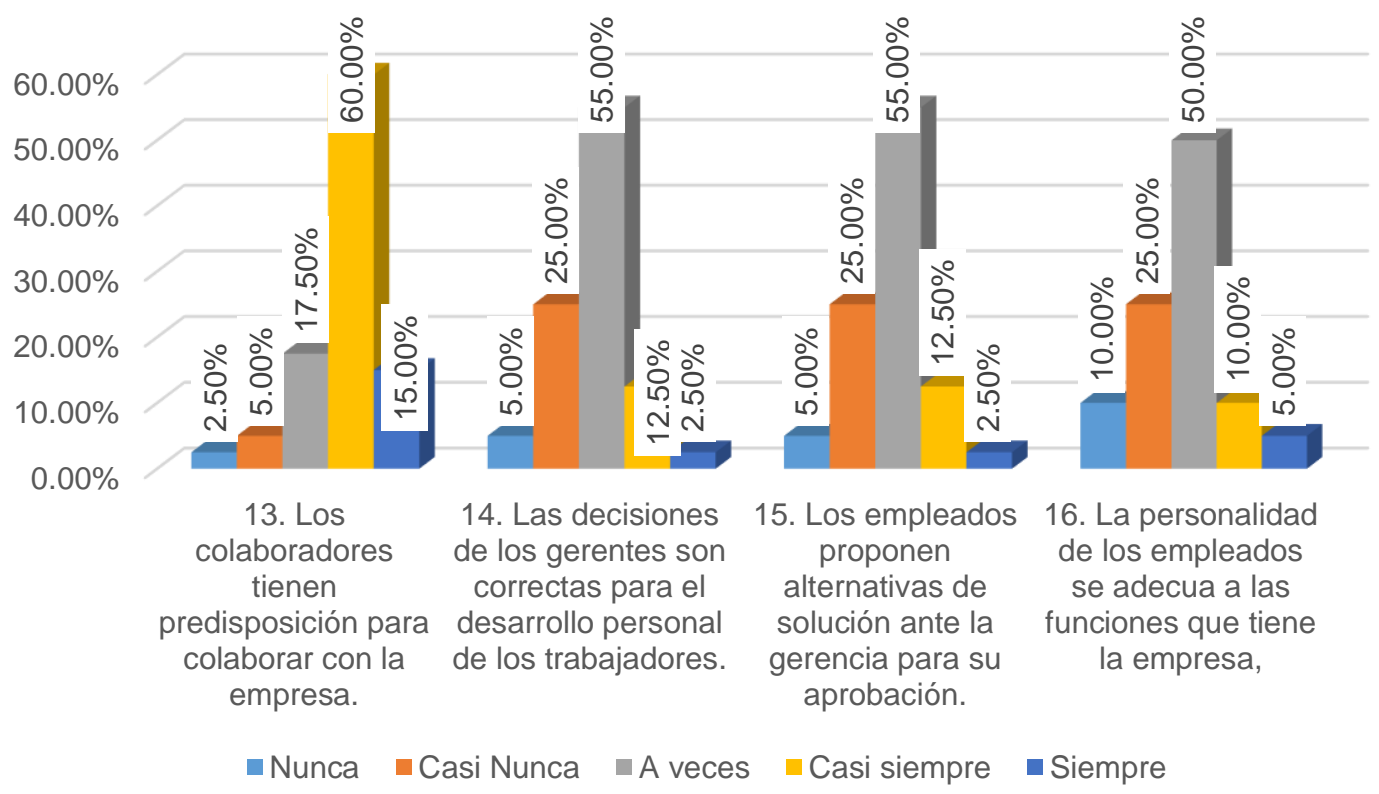

Figura 3. Frecuencias de la sub categoría empowerment (Fuente: elaboración propia)

En el caso del empowerment, se analizaron los factores más críticos, 95\% reveló no tener una buena imagen de la empresa por el poco apoyo que brinda a los trabajadores, $95 \%$ indica que los trabajadores no se integran adecuadamente y no trabajan en equipo, $95 \%$ reveló que la empresa no invierte en realizar actividades que mejores el sentido de pertenencia de los trabajadores, y, 95\% evidencia que la empresa no muestra interés en que los empleados participen de eventos relacionados con la compañía.

Para finalizar el análisis cuantitativo, se realizó el diagrama de Pareto. Siguiendo los lineamientos de la teoría, se trata de una herramienta con enfoques de calidad, y cuyo fundamento gráfico busca plasmar los datos más prioritarios. Con un criterio de elección entre el $20 \%$ y $80 \%$ (Universidad de Vigo, 2006), como se observa en la figura 4. 


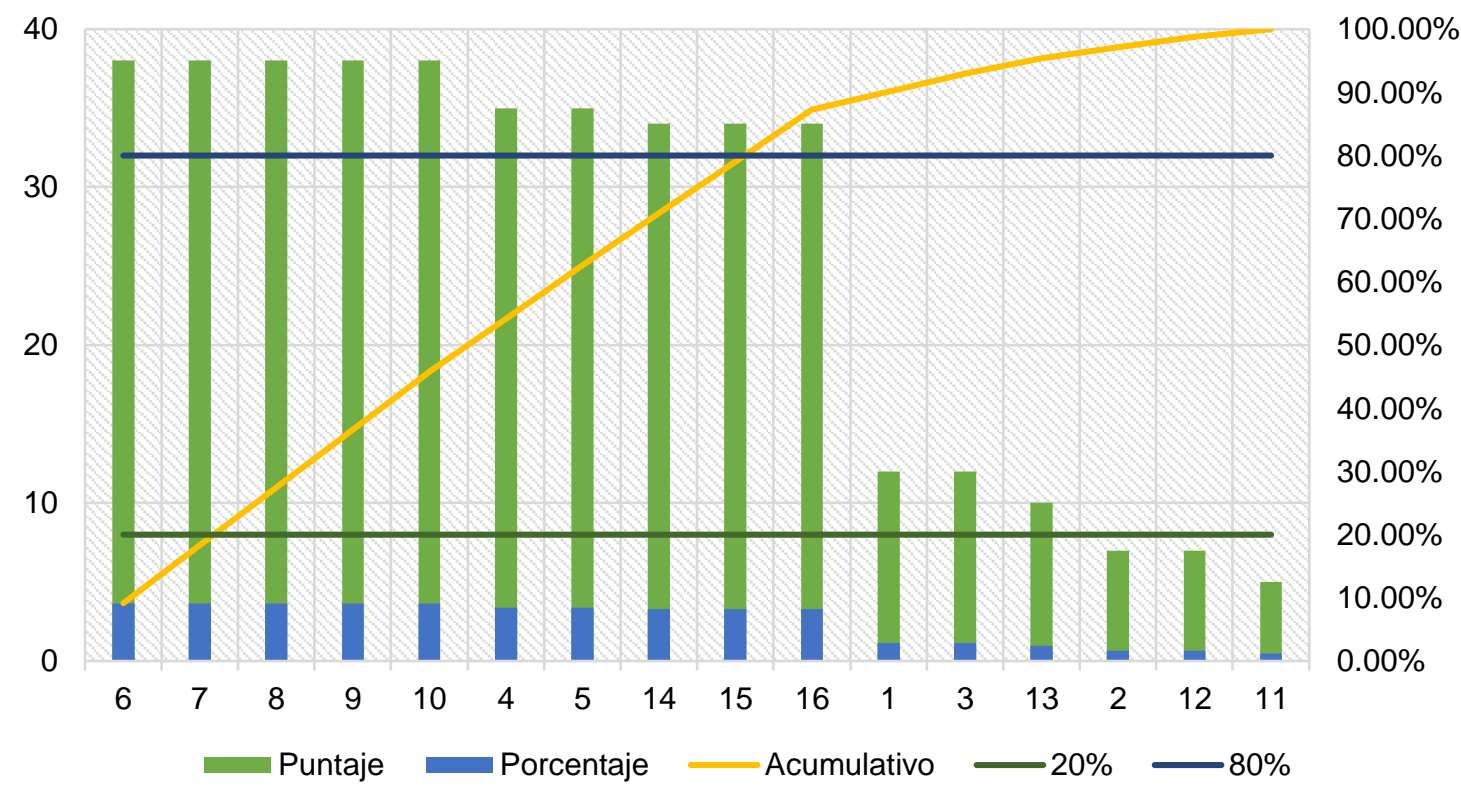

Figura 4. Pareto de la categoría comunicación interna Fuente: elaboración propia

Los problemas más urgentes de solución fueron el ítem 6, la comunicación para resolver problemas de gestión y el ítem 7, la imagen que tiene la compañía.

A continuación, se plantea el uso de datos de carácter cualificado, donde se plasman a partir de la experiencia acumulada durante las etapas de trabajo (Monjé, 2011). El análisis cualitativo se procesó en base a cada una de las subcategorías y cada una de las preguntas de la guía de entrevista formulada a expertos, y fueron procesada con el software Altas ti, resultando en la formación de redes de ideas fuerza, la sub categoría proceso administrativo, como lo muestra la figura 5, arrojó los siguientes resultados. 


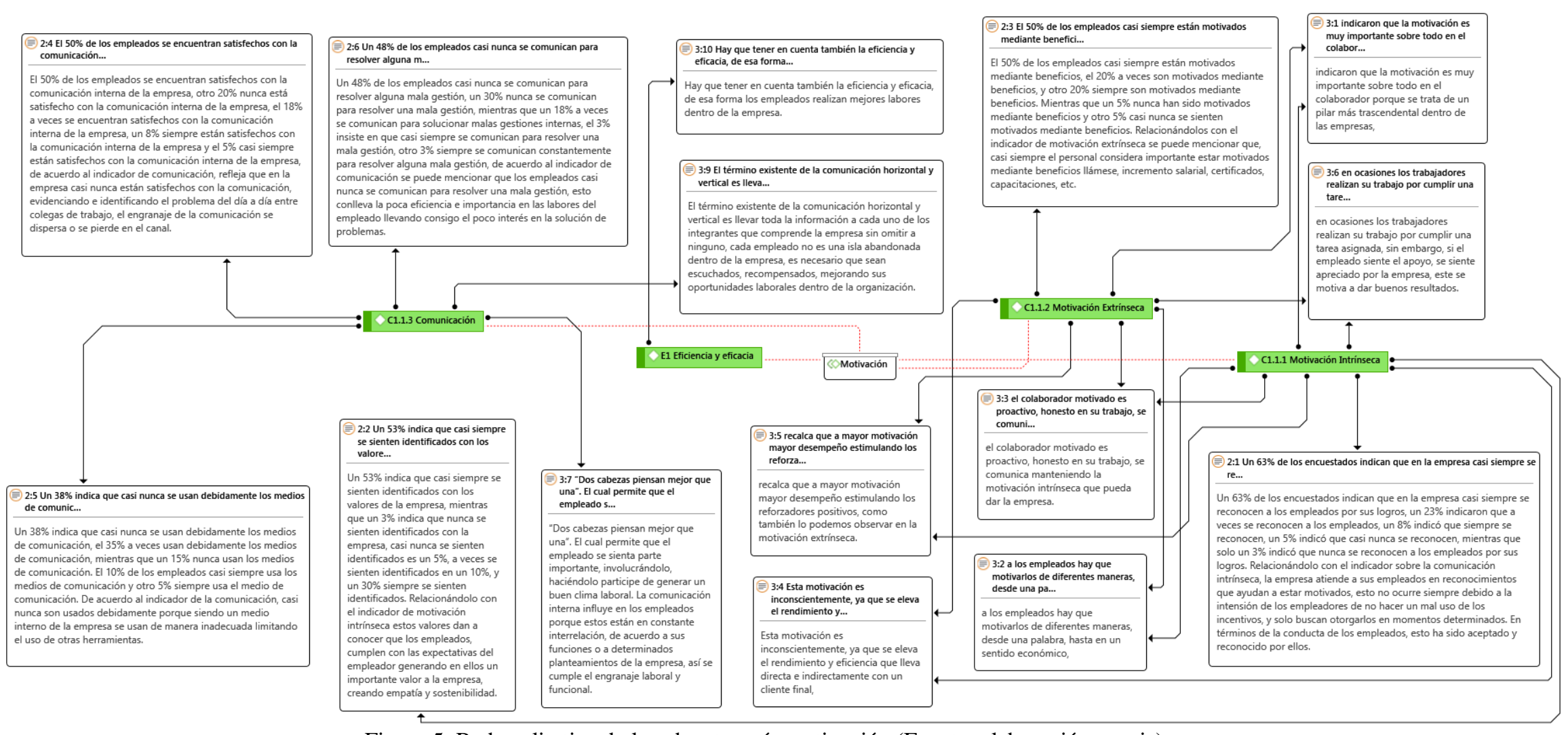

Figura 5. Red cualitativa de la sub categoría motivación (Fuente: elaboración propia) 
Los resultados obtenidos destacan la motivación intrínseca como parte de la motivación del empleado y le da un valor transcendental en la organización, así mismo, remarca el rendimiento y eficiencia como parte del valor que tiene el trabajador al momento de tener contacto con el cliente. Y la motivación extrínseca, como sentido económico del empleado y la forma en la que se identifica con la compañía. La comunicación horizontal y vertical como método para integrar a los trabajadores y lograr un sentido de pertenencia.

En segundo lugar, la sub categoría identidad corporativa, como lo muestra la figura 6, arrojó los siguientes resultados. 


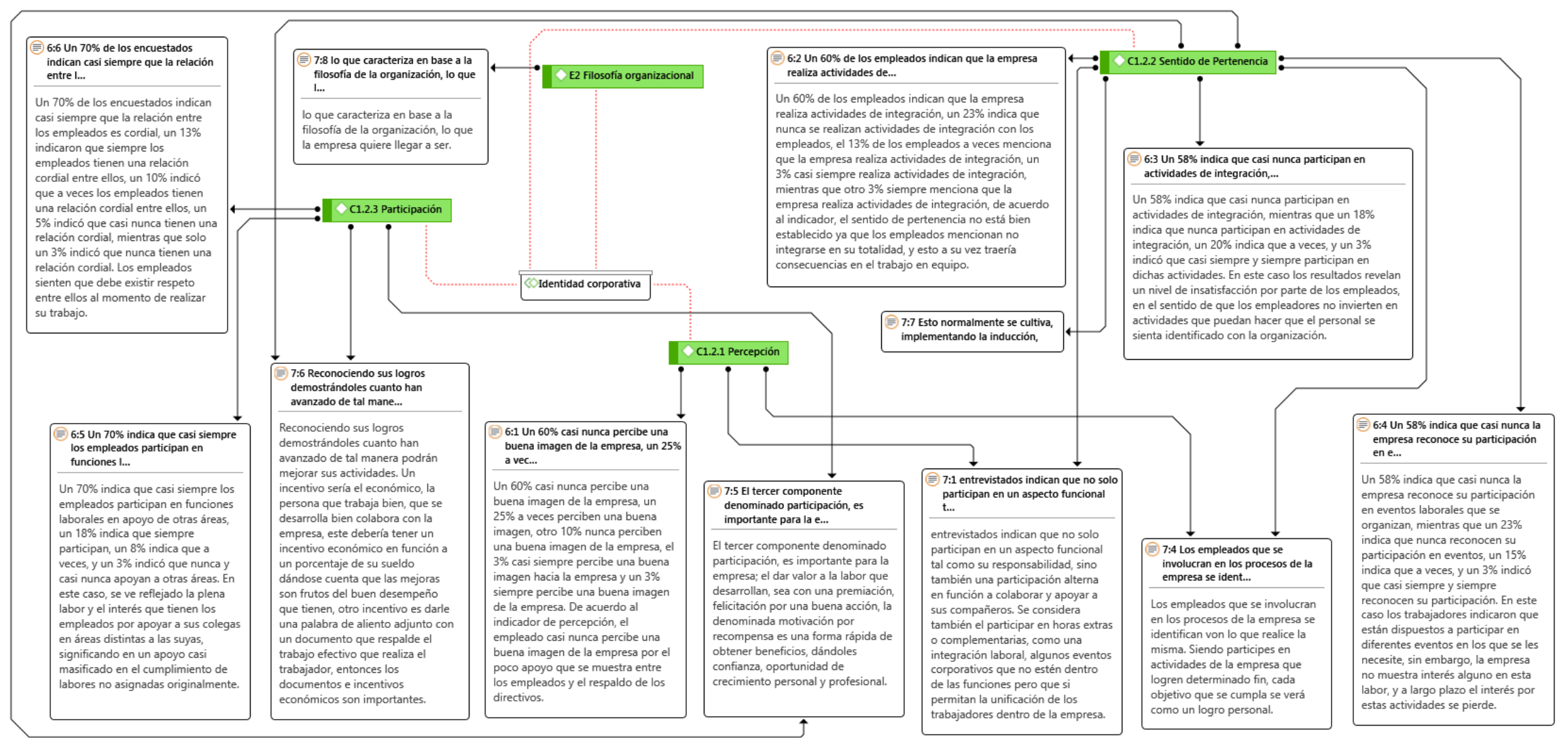

Figura 6. Red cualitativa de la sub categoría identidad corporativa (Fuente: elaboración propia) 
Este apartado indicó que el sentido de pertenencia es un aspecto fundamental y tiene como responsabilidad lograr la participación de los empleados en las actividades de la empresa, como función de la colaboración y apoyo en actividades adicionales de la compañía. Así mismo, la integración laboral se logra mediante eventos corporativos y alienten el trabajo colectivo de las personas. La inducción se caracteriza por efectos de capacitación y dirección hacia los objetivos. Y como componente de participación, desarrolla valor mediante sentidos de recompensa y unificación.

En tercer lugar, la sub categoría empowerment, como lo muestra la figura 7, arrojó los siguientes resultados. 


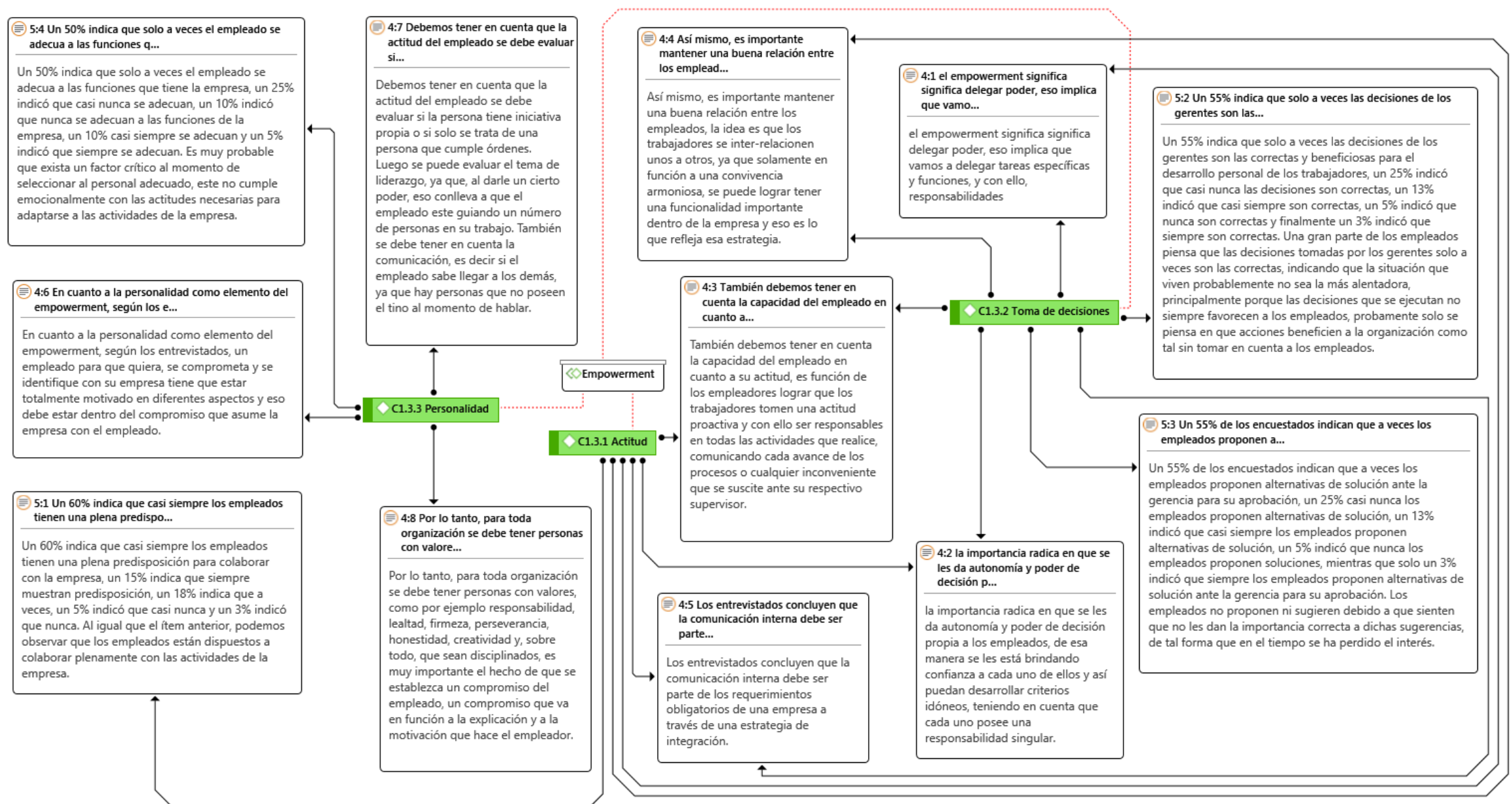

Figura 7. Red cualitativa de la sub categoría empowerment (Fuente: elaboración propia) 
En este apartado, da importancia a la delegación de poder, que implica responsabilidades en los trabajadores, así mismo, se identifican con la empresa desde el sentido de la motivación y aspectos internos que asume el empleado. Y el tema de los valores, como ejemplo de responsabilidad, lealtad y firmeza.

El análisis mixto se procesó utilizando los resultados cuantitativos y cualitativos, en cada sub categoría, su importancia radica en la unión de dos metodologías, y se realiza dentro de una sistematización conceptual y numérica (Ruiz, 2011). Plasmada en la formación de redes de ideas fuerza, la sub categoría motivación, como lo muestra la figura 8, arrojó los siguientes resultados. 


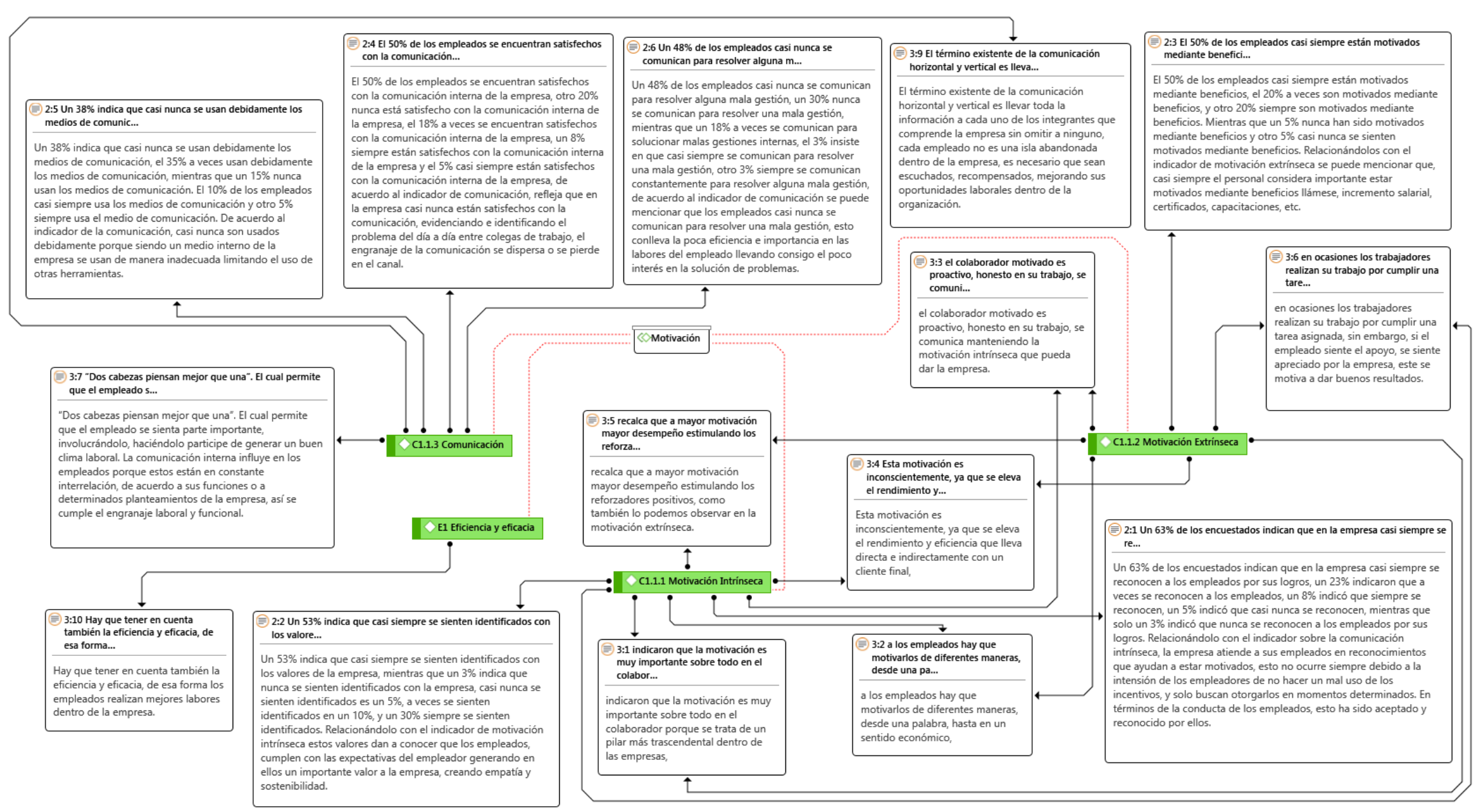

Figura 8. Red mixta de la motivación (Fuente: elaboración propia) 
El análisis mixto enmarca la idea de la comunicación, que permite al empleado se siente involucrado en las labores de la empresa y se genere un buen clima laboral, como $88 \%$ indica, no se encuentra satisfecho con la comunicación en la compañía. Así mismo, la comunicación horizontal y vertical lleva la información a todos los integrantes, ya que como $88 \%$ indicó, los medios de comunicación no funcionan y son importantes para la organización.

Luego, la sub categoría identidad corporativa, como lo muestra la figura 9, arrojó los siguientes resultados. 


\begin{tabular}{l} 
e) 6:5 Un 70\% indica que casi siempre los \\
empleados participan en funciones I... \\
\hline Un $70 \%$ indica que casi siempre los \\
empleados participan en funciones \\
laborales en apoyo de otras áreas, un \\
$18 \%$ indica que siempre participan, un \\
$8 \%$ indica que a veces, y un $3 \%$ indicó \\
que nunca y casi nunca apoyan a otras \\
áreas. En este caso, se ve reflejado la \\
plena labor y el interés que tienen los \\
empleados por apoyar a sus colegas en \\
áreas distintas a las suyas, significando en \\
un apoyo casi masificado en el \\
cumplimiento de labores no asignadas \\
oriqinalmente.
\end{tabular}

\section{7:6 Reconociendo sus logros
demostrándoles cuanto han avanzado de}

Reconociendo sus logros

demostrándoles cuanto han avanzado de

tal manera podrán mejorar sus

actividades. Un incentivo sería el

económico, la persona que trabaja bien,

que se desarrolla bien colabora con la

empresa, este debería tener un incentivo

económico en función a un porcentaje

de su sueldo dándose cuenta que las

mejoras son frutos del buen desempeño

que tienen, otro incentivo es darle una

palabra de aliento adjunto con un

documento que respalde el trabajo

efectivo que realiza el trabajador,

entonces los documentos e incentivos

económicos son importantes.

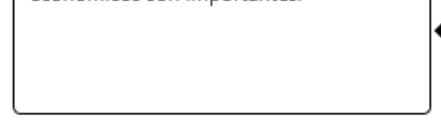

7:7 Esto normalmente se cultiva, implementando la inducción,

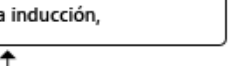

\section{(-) $6: 6$ Un $70 \%$ de los encuestados indican casi \\ siempre que la relación entre l...}

Un 70\% de los encuestados indican casi siempre que la relación entre los empleados es cordial, un $13 \%$ indicaron que siempre los empleados tienen una relación cordial entre ellos, un $10 \%$ indicó que a veces los empleados tienen una relación cordial entre ellos, un $5 \%$ indicó que casi nunca tienen una relación cordial, mientras que solo un $3 \%$ indicó que nunca tienen una relación cordial. Los empleados sienten que debe existir respeto entre ellos al momento de realizar su trabajo.

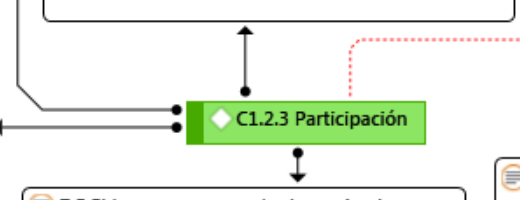

$7: 5$ El tercer componente denominado
participación, es importante para la e...

El tercer componente denominado participación, es importante para la empresa; el dar valor a la labor que desarrollan, sea con una premiación elicitación por una buena acción, la denominada motivación por recompensa es una forma rápida de obtener beneficios, dándoles confianza, oportunidad de crecimiento personal y profesional.

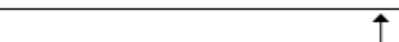

7:8 lo que caracteriza en base a la filosofia de la organización, lo que

lo que caracteriza en base a la filosofía de la organización, lo que la empresa quiere llegar a ser.

\begin{tabular}{l} 
6:2 Un 60\% de los empleados indican que la empresa realiza \\
actividades de... \\
\hline Un $60 \%$ de los empleados indican que la empresa realiza \\
actividades de integración, un $23 \%$ indica que nunca se realizan \\
actividades de integración con los empleados, el $13 \%$ de los \\
empleados a veces menciona que la empresa realiza actividades \\
de integración, un $3 \%$ casi siempre realiza actividades de \\
integración, mientras que otro $3 \%$ siempre menciona que la \\
empresa realiza actividades de integración, de acuerdo al \\
indicador, el sentido de pertenencia no está bien establecido ya \\
que los empleados mencionan no integrarse en su totalidad, y \\
esto a su vez traería consecuencias en el trabajo en equipo.
\end{tabular}

6:3 Un 58\% indica que casi nunca participan en actividades de integración,...

Un $58 \%$ indica que casi nunca participan en actividades de integración, mientras que un $18 \%$ indica que nunca participan en actividades de integración, un $20 \%$ indica que a veces, y un $3 \%$ indico que casi siempre y siempre participan en dichas actividades. En este caso los resultados revelan un nivel de insatisfacción por parte de los empleados, en el sentido de que los empleadores no invierten en actividades que puedan hacer que el personal se sienta identificado con la organización

\section{CIdentidad corporativa}

E2 Filosofía organizacional

(-) 6:1 Un 60\% casi nunca percibe una buena imagen de la empresa, un $25 \%$ a vec...

Un 60\% casi nunca percibe una buena imagen de la empresa, un $25 \%$ a veces perciben una buena imagen otro $10 \%$ nunca perciben una buena imagen de la empresa, el $3 \%$ casi siempre percibe una buena imagen hacia la empresa y un $3 \%$ siempre percibe una buena imagen de la empresa. De acuerdo al indicador de percepcion, el empleado casi nunca percibe una buena imagen de la empresa por el poco apoyo que s muestra entre los empleados y el respaldo de los directivos

\section{7:1 entrevistados indican que no solo participan en un aspecto funcionat} entrevistados indican

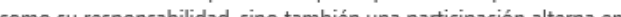
función a colaborary apoyar a sus compañeros Se considera también el participar en horas extras o complementarias, como una integración peripla pero que si permitan la unificación de los trabajadores dentro de lancio empresa.

\section{6:4 Un $58 \%$ indica que casi nunca la empresa reconoce su \\ participación en e.}

Un $58 \%$ indica que casi nunca la empresa reconoce su participacion en eventos laborales que se organizan, mientras que un $23 \%$ indica que nunca reconocen su participación en eventos, un $15 \%$ indica que a veces, y un $3 \%$ indicó que casi siempre y slempre reconocen su participación. En este caso los trabajadores indicaron que están dispuestos a participar en diferentes eventos en los que se les necesite, sin embargo, la empresa no muestra interés alguno en esta labor, $y$ a largo azo el interés por estas actividades se pierde.

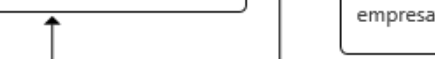

Figura 9. Red mixta de la identidad corporativa (Fuente: elaboración propia) 
En este caso se considera importante la imagen que tienen los trabajadores por la compañía, como indica 95\% no perciben una buena imagen y no se identifican con ella. También, 94\% resalta la idea de la participación en eventos corporativos, como un gran interés que debería tener la compañía. Así mismo, indica que la integración de los empleados es importante para el logro personal de los mismo, como lo indica $94 \%$ no participan en eventos de integración.

Finalmente, la sub categoría empowerment, como lo muestra la figura 10, arrojó los siguientes resultados. 


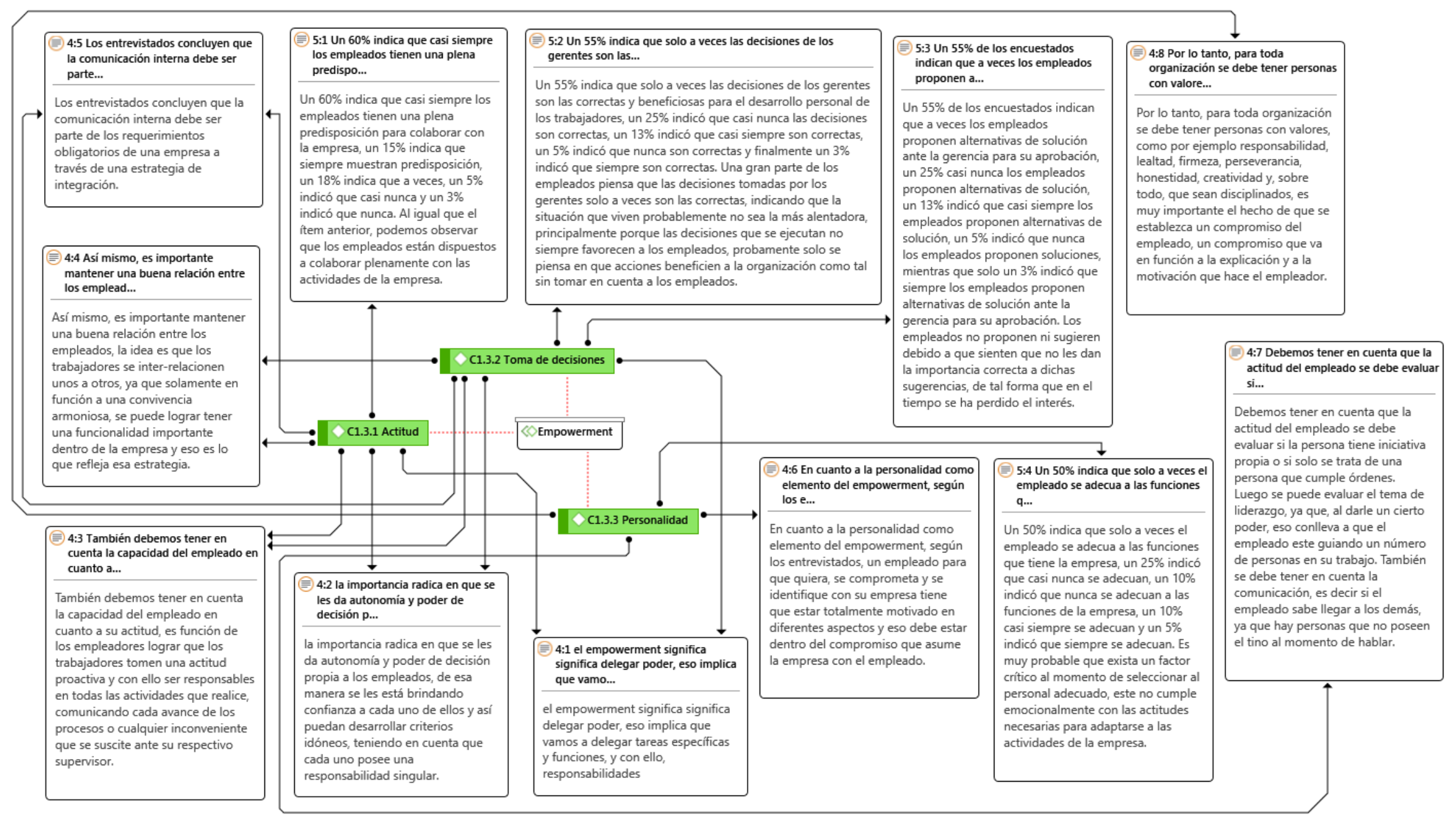

Figura 10. Red mixta de empowerment (Fuente: elaboración propia) 
En este caso, destaca la actitud del empleador para lograr que los trabajadores realicen con éxito sus responsabilidades, tanto en temas, de desarrollo personal y de actividades, tal y como expresa $85 \%$ de encuestados, que indica que los gerentes no toman decisiones que ayuden al desarrollo de los empleados. Así mismo, $85 \%$ indica que no proponen alternativas de solución a la gerencia. También desataca el tema de la personalidad, que en los empleados ayuda a mejorar la integración y el cumplimiento de objetivos. Tanto como $85 \%$ indicó, los trabajadores no cumplen con sus funciones en favor de sus objetivos.

\section{Propuesta}

Tal como se planteó en el objetivo inicial, se propone mejorar la comunicación interna, y, como eje central, resolver los problemas de comunicación, la poca identidad corporativa, y, la motivación del personal. Para dicho propósito, se buscó aplicar la estrategia del employer engagement, el cual permite establecer mecanismos para lograr el bienestar emocional de los empleados y busca, en las organizaciones, que se generen beneficios mutuos. Como parte del desarrollo empresarial, permite encontrar cualidades en los empleados y maximizarlas. Así mismo, acelera el proceso de integración, permitiendo a los trabajadores ser parte de actividades que desarrollan su bienestar social (Mitchell, 2016).

Utilizando el enfoque propuesto por el employer engagement, para mejorar la comunicación, se utiliza el Branding Employee, un proceso para mejorar el compromiso y establecer mecanismos para mejorar las relaciones interpersonales (Aguado \& Jiménez, 2009).

El objetivo es buscar un ambiente de trabajo agradable, que permite el desarrollo personal y el compromiso que el empleado tiene con la organización.

El Branding Employee desarrolla la confianza de los empleados, para esto se plante el siguiente esquema de trabajo, como se observa en la figura 11. 


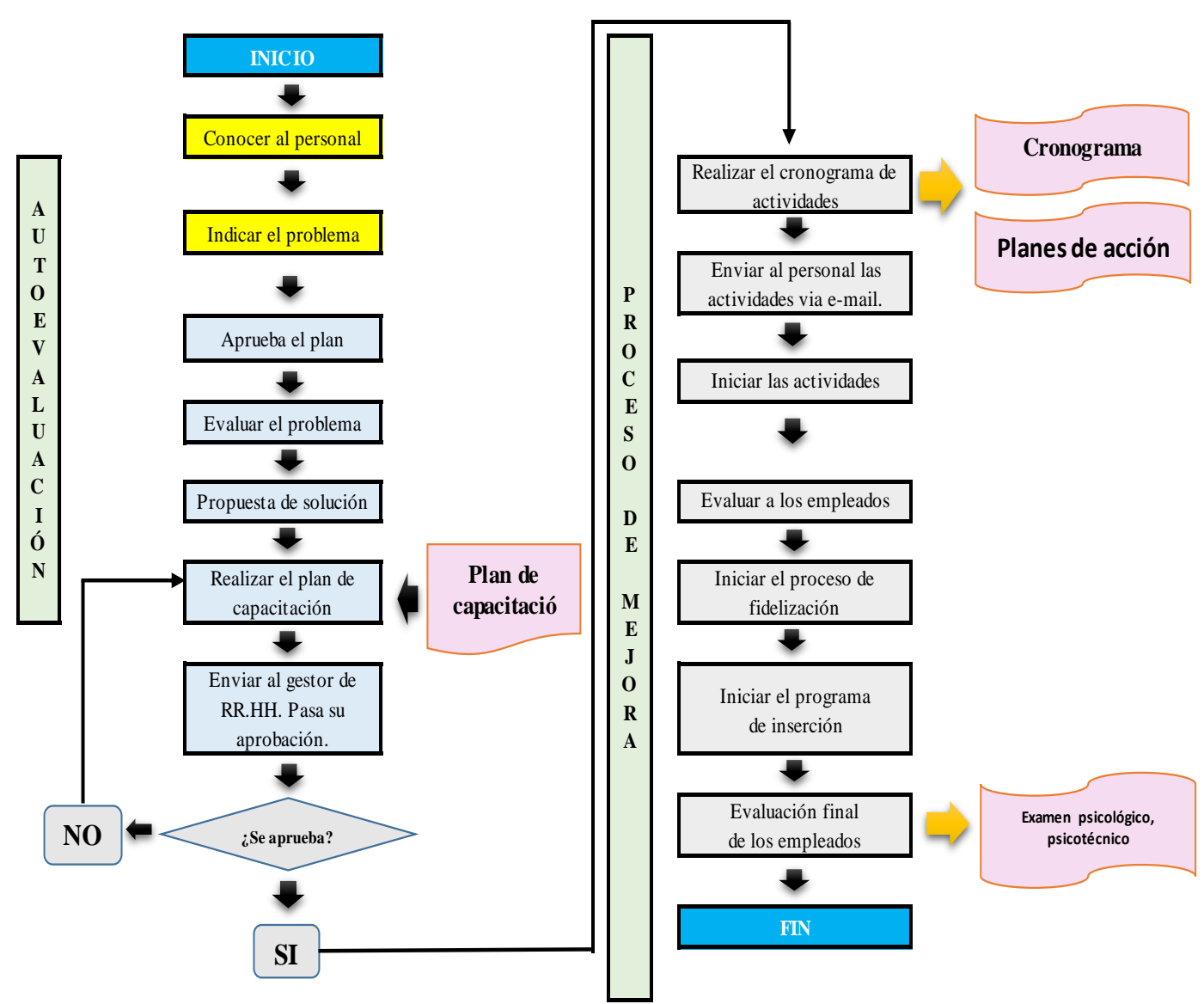

Figura 11. Flujograma de actividades del Branding Employee (Fuente: elaboración propia)

Por otro lado, para lograr un recurso humano altamente calificado y motivado, se plantea el uso de los recursos humanos 2.0, que basa su aplicación en el reconocimiento y el uso de la información como estrategia para cumplir objetivos.

Como agente de cambio, se establece la tecnología como principal fuente para acercarse a los empleados. Al mismo tiempo plantea mejores mecanismos para contratar personal capacitados y mejorar el que ya se tiene.

La figura 12 plantea las actividades a cumplir para desarrollar el esquema de los recursos humanos 2.0. 


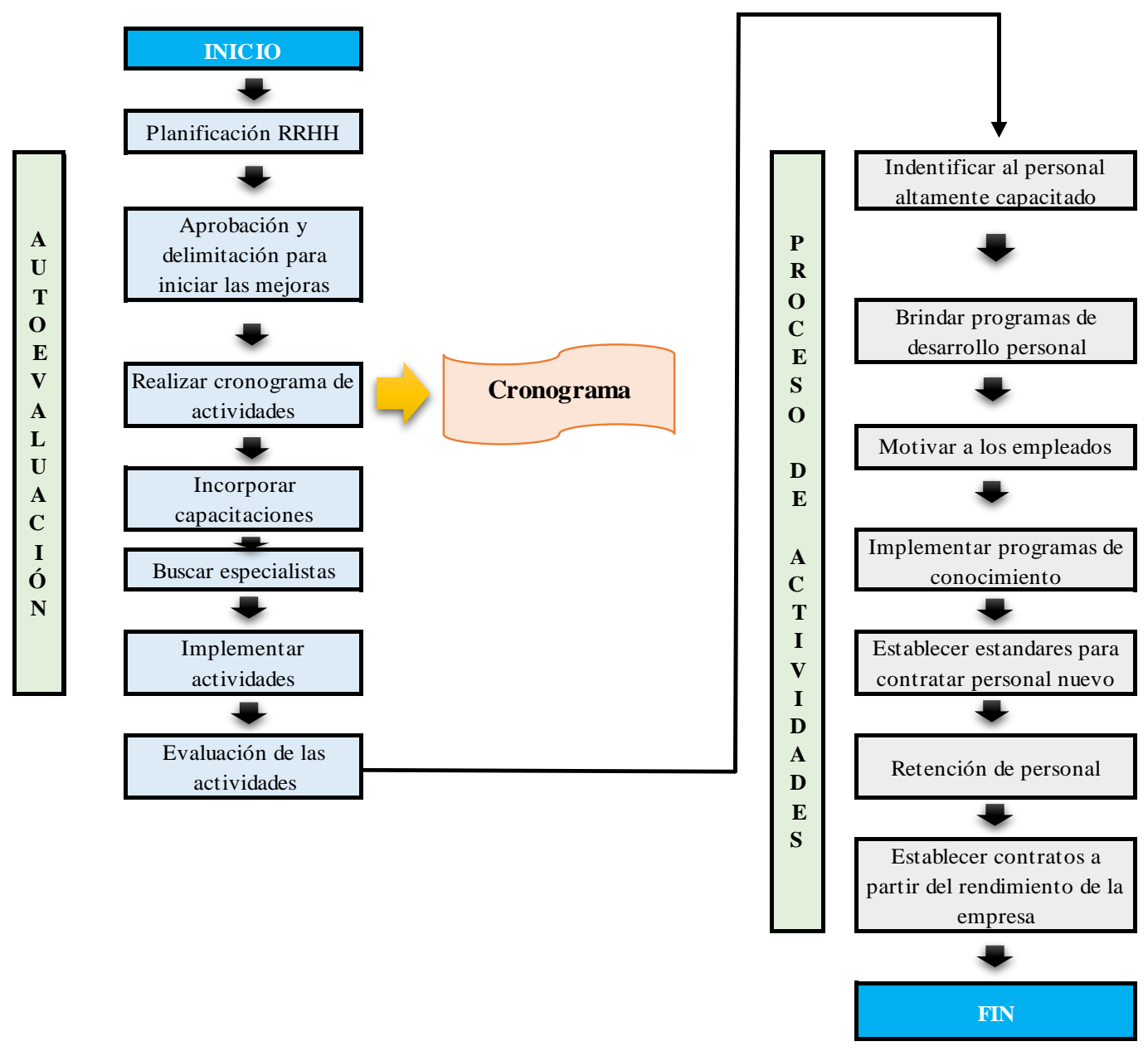

Figura 12. Flujograma de actividades de los RR.HH. 2.0 (Fuente: elaboración propia)

Finalmente, para poder incrementar la identidad corporativa y el sentido de pertenencia de los trabajadores, se optó por realizar una capacitación de inteligencia emocional.

La misma que se constituye como la adaptación de habilidades emocionales y psicológicas, que se manejan dentro de un proceso de empatía hacia sí mismo y hacia los demás. Comprendiendo procesos de análisis y simpatía entre las personas (Goleman, 1996).

Dicha capacitación abarca los temas de autoconciencia, autorregulación, motivación, empatía y habilidades sociales.

Para llevarlo a cabo se plantean actividades expuestas en la figura 13. 


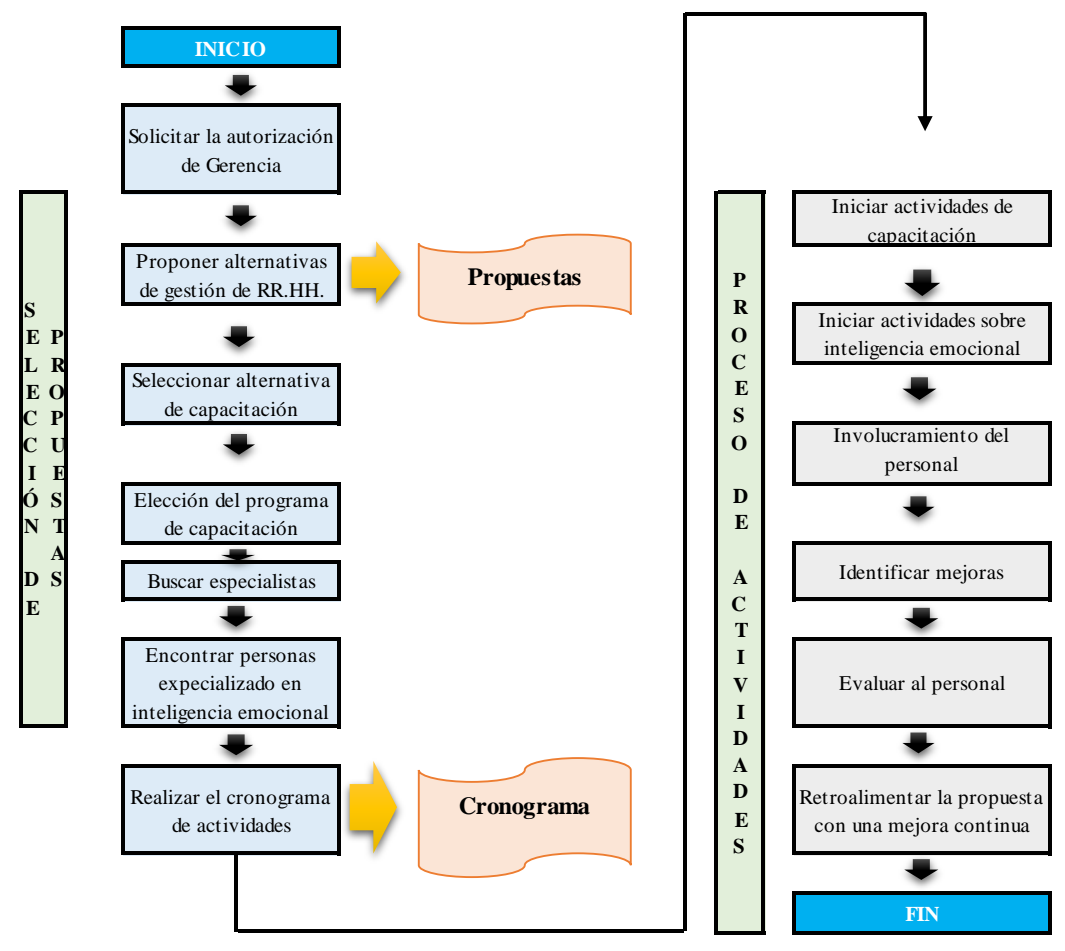

Figura 13. Flujograma de actividades para capacitación (Fuente: elaboración propia)

\section{Conclusiones}

Se decidió implementar como propuesta el employer engagement, para la mejora de la comunicación interna y el compromiso del empleado, la cual resultará en procesos eficientes para lograr metas y objetivos.

Así mismo, para un mejor desarrollo de la investigación se analizan los aspectos de la motivación, la identidad corporativa y el empowerment, todas sub categorías de la comunicación interna. Su estudio indicó que los problemas más importantes a corregir fueron la poca comunicación en la organización, la identificación con la empresa, y, la motivación del personal.

Además, como factores de mayor incidencia, se encontró falta de integración entre los empleados, poco reconocimiento por parte de los empleadores, mal uso de los medios de comunicación, falta de propuestas y proyectos de integración.

La alternativa de solución influye en el empleado, de tal forma que mejora la comunicación interna y sus diferentes estrategias. En el caso de los recursos humanos 2.0, influye en los trabajadores, aumentando la motivación y capacitándolos para tareas más complicadas, y la capacitación en inteligencia emocional, que desarrolla la identidad corporativa y el compromiso. 


\section{Bibliografía}

Aguado, M., \& Jiménez, A. (2009). Employer Branding La gestión de las marcas para atraer y retener el talento. Córdoba: Almuzara.

Armas, J. (2014). Comunicacion interna y clima laboral. Guatemala: Universidad Rafael Landívar.

Berceruelo, B. (2011). Comunicacion interna en la empresa. Valencia, España: Wolters Kluwer España.

Chiavenato, I. (2014). Introduccion a la teoría general de la administración. México, D.F.: Mc Graw Hill Education.

Condor, H. (2018). La gestión de la comunicación interna y el clima organizacional en el sector público. SciELO Analytics, 25-34.

Cukier Mariana. (2011, noviembre 1). Mejorar la comunicación interna de la empresa mediante una red de facilitadores. Recuperado de https://www.gestiopolis.com/mejorarcomunicacion-interna-empresa-mediante-red-facilitadores/

Currás Pérez, R. (2010). Identidad e imagen corporativas: revisión conceptual e interrelación. Teoría y Praxis, 9-34.

Durán Rodríguez Manuela. (2009, marzo 24). Sistema de comunicación interna en las organizaciones. Recuperado de https://www.gestiopolis.com/sistema-comunicacioninterna-organizaciones/

Evans, K., Larson, A., Mwangi, E., Cronkleton, P., Maravanyika, T., Hernández, X., . . . Banana Abwoli. (2015). Guía práctica de manejo adaptativo y colaborativo (ACM) y mejora de la participación de las mujeres. Indonesia: CIFOR.

Fernández , J., \& Puente Ferreras, A. (2009). La noción de campo en Kurt Lewin y Pierre Bourdieu: Un análisis comparativo. Revista Española de investigaciones sociológicas, 33 53.

French, M. (2016). 5 pasos para crear Dashboards Efectivos. Argentina: Sixtina Consulting Group.

Garcia, J. (2017). Valoracion del aporte de la comunicacion interna y factores relacionados. Investigacion en organizaciones privadas de la ciudad de Lima durante el 2017. Lima: PUCP.

Goleman, D. (1996). Inteligencia emocional. Barcelona: KAIROS.

Gutierez Cottle, C. M. (2017). La importancia de la comunicacion interna en organizaciones del tercer sector: Caso enseñe Perù. Lima: Universidad de Lima.

Hernández Sampieri, R., Fernandez Collado, C., \& Baptista Lucio, M. (2014). Metodología de la Investigación. México: Ms Graw Hill Education.

Herzberg, F. (1968). One more time: How do you motivate employees? Bussines Review, 01-10.

Hurtado, J. (2000). Metodologia de la Investigacion Holistica. Caracas: Sypal.

Iturrioz Aizpuru, J. (2017). El liderazgo en el siglo XXI Coaching, equipos, clientes y resultados. Grupo Planeta.

Mitchell, M. (2016). Techniques Employer Engagement Indutry's Role in CTE Program Delivery. Estados Unidos: ACTE.

Monjé Álvarez, C. A. (2011). Metodología de la investigación cuantitativa y cualitativa. Neiva: Universidad surcolombia.

Moreno, J. (2015). La comunicación interna: Una herramienta al servicio de las organizaciones. Barcelona-España: Universidad de Barcelona. 
Peiro, J., \& Bresó, I. (2012). La comunicación en las organizaciones: Una aproximación desde el modelo de análisis multifacético para la gestión y la intervención organizacional (modelo Amigo). Redalyc, 41-70.

Pineda, A. (2007). Compromiso organizacional de los trabajadores de una Universidad Pública. Redalyc, 30-37.

Porter, L., Hackman, J., \& Lawler, E. (1975). Behavior in Organizations. España: McGraw-Hill.

Preciado, A., \& Etayo, C. (2014). Influencia del estilo directivo en la comunicacion interna de las organizaciones: Una aplicacion a las agencias de publicidad.

Rodríguez, E. (7 de Diciembre de 2018). La teoría de las necesidades de McClelland. Obtenido de La mente es maravillosa: https://lamenteesmaravillosa.com/la-teoria-de-las-necesidadesde-mcclelland/

Ruiz Medina, M. (2011). Politicas públicas en salud y su impacto en el seguro popular en Culiacán, Sinaloa, México. Sinaloa: Universidad Autónoma de Sinaloa.

Terry, W. (1996). Manual del Empowerment. España: Ediciones gestion 2000.

Tessi, M. (2013). Comunicacion interna en la práctica: Siete premisas para la comunicación en el trabajo. Granica, Buenos Aires: Ediciones Granica S.A.

Universidad de Vigo. (2 de Mayo de 2006). Diagrama de Pareto. Recuperado el 18 de Junio de 2019, de Gestión de la calidad, la seguridad y el medio ambiente: http://gio.uvigo.es/asignaturas/gestioncalidad/GCal0405.DiagramaPareto.pdf 